\title{
Controversias en cirugía: Erradicación del Helicobacter pylori ¿Terapia a todos o según indicaciones usuales?
}

\author{
Controversies in surgery: Eradication of Helicobacter pylori. Therapy for all \\ or according to usual indications?
}

\author{
Esteban Isaza-Gómez (D), Manuel Santiago Ángel-González $\mathbb{D}$, Maribel Ocampo-Muñoz (D), \\ Camilo Andrés Díaz-QuinteroDi, Isabel Cristina Molina-Céspedes (D), \\ María Antonia Velásquez-Martínez DD, Pablo Posada-Moreno $\mathbb{D}$, Santiago Salazar-OchoaD
}

Médicos, residentes de Cirugía General, Universidad Pontificia Bolivariana, Medellín, Colombia.

\section{Resumen}

La infección por Helicobacter pylori (H. pylori), es la infección bacteriana crónica más frecuente de la raza humana, afecta al $50 \%$ de la población mundial y, por lo menos, al 80 \% de la población colombiana. Esta bacteria es reconocida desde hace más de 15 años como un carcinógeno tipo I. De acuerdo con las indicaciones del Consenso de "Maastricht V" esta infección debe ser buscada y tratada en los pacientes con úlcera péptica activa, linfoma MALT (por sus siglas en inglés, mucosa associated lymphoid tissue), cáncer gástrico temprano, púrpura que presenten síntomas dispépticos crónicos y usuarios crónicos de AINES.

Debido al papel que tiene en la fisiopatología del cáncer gástrico, nace la iniciativa de realizar una búsqueda activa del $H$. pylori y erradicarlo en todas las personas, incluyendo aquellas asintomáticas en países con alta incidencia de esta neoplasia.

Existen diversas publicaciones alrededor del mundo que así lo sugieren, mostrando resultados con impacto positivo en el curso y progresión de la enfermedad, sobre todo en las etapas más tempranas de la infección.

Sin embargo, otros autores resaltan la creciente problemática de la resistencia bacteriana, y demuestran que el peso estadístico y los diferentes análisis de los estudios disponibles en la actualidad tienen poca validez para dar una recomendación extendida al paciente asintomático. Se cuestiona que tal vez, estamos utilizando las estrategias inadecuadas para manejar una situación de salud pública, ya que estamos enfocados en impactar a cada individuo con terapias antibióticas complejas, en vez de a la población en general con políticas de salud pública.

Palabras clave: Helicobacter pylori; neoplasias gástricas; úlcera gástrica; linfoma del tejido linfático asociado a mucosas; antibacterianos; farmacorresistencia bacteriana.

Fecha de recibido: 11/2/2020 - Fecha de aceptación: 24/5/2020

Correspondencia: Esteban Isaza-Gómez, Universidad Pontificia Bolivariana, Calle 78B \# 72 A - 109, Medellín, Colombia.

Teléfono: (57 4) 4488388

Correo electrónico: residentescirugiaupb@gmail.com

Citar como: Isaza-Gómez E, Ángel-González MS, Ocampo-Muñoz M, Díaz-Quintero CA, Molina-Céspedes IC, Velásquez-Martínez MA, et al. Controversias en cirugía: Erradicación del Helicobacter pylori ¿Terapia a todos o según indicaciones usuales? Rev Colomb Cir. 2020;35:665-74. https://doi.org/10.30944/20117582.563

Este es un artículo de acceso abierto bajo una Licencia Creative Commons - BY-NC-ND https://creativecommons.org/licenses/by-ncnd/4.0/deed.es 


\begin{abstract}
Helicobacter pylori (H. pylori) infection is the most frequent chronic bacterial infection in humans, affecting $50 \%$ of the world population, and at least $80 \%$ of the Colombian population. This bacteria has been recognized for more than $\mathrm{I} 5$ years as a type I carcinogen. According to the indications of the "Maastricht V" consensus, this infection should be sought and treated in patients with: active peptic ulcer, MALT lymphoma (for its acronym Mucosa associated lymphoid tissue), early gastric cancer, purpura who present with chronic dyspeptic symptoms and chronic users of NSAIDs. Due to the role it plays in the pathophysiology of gastric cancer, the initiative was born to carry out an active search for $\mathrm{H}$. pylori and eradicate it in all people, including those asymptomatic in countries with a high incidence of this neoplasia.

There are various publications around the world that suggest the effectiveness of this treatment and the positive impact on the course and progression of the disease, especially in the earliest stages of the infection, since the more advanced stages have less encouraging results regarding progression to malignancy. However, other authors highlight the growing problem of bacterial resistance that we are currently facing and demonstrate that the statistical weight and the different analyzes of the currently available studies have little validity to give an extended recommendation to the asymptomatic patient. It is suggested that perhaps inappropriate strategies to manage this public health situation are being used, since we are focused on impacting each individual with complex antibiotic therapies, instead of the general population with public health policies.
\end{abstract}

Keywords: Helicobacter pylori; stomach neoplasms; stomach ulcer; mucosa-associated lymphoid tissue lymphoma; anti-bacterial agents; drug resistance, bacterial.

\section{Introducción}

Actualmente la infección por el Helicobacter pylori (H. pylori), es la infección bacteriana crónica más frecuente de la raza humana ${ }^{1}$. Desde su descubrimiento en la década de los ochenta, se ha aprendido mucho de este microorganismo Gram negativo, en forma de espiral y flagelado. En 1994 se reconoció como causal directo de úlceras pépticas gástricas y duodenales, además, se estableció como un carcinógeno tipo I para el adenocarcinoma gástrico ${ }^{2}$. Se conoce que el IOo \% de los infectados por este germen tienen algún grado de gastritis crónica, y de estos hasta el $20 \%$ tienen algún otro tipo de gastropatía asociada, como enfermedad ulcerosa, atrofia gástrica, linfoma tipo MALT (por sus siglas en inglés, mucosa associated lymphoid tissue), pólipos hiperplásicos gástricos, enfermedades hematológicas como púrpura trombocitopénica inmune, anemia por deficiencia de vitamina BI2 o ferropénica, y hasta el $3 \%$ de los infectados desarrollarán un adenocarcinoma gástrico en relación directa a la infección ${ }^{3,4}$.
El H. pylori afecta al $50 \%$ de la población mundial y, por lo menos, al 8o \% de la población colombiana ${ }^{5,6}$. Es catalogado actualmente como la principal causa etiológica, combinada con factores genéticos y dietarios, para el desarrollo del cáncer gástrico (riesgo atribuible de hasta un $90 \%{ }^{4}$, el cual, en Colombia, es la primera causa de muerte por cáncer en los hombres y la tercera en las mujeres ${ }^{6}$. Por todo esto, el $H$. pylori representa un riesgo importante en las zonas de alta incidencia de cáncer gástrico.

Debido a las patologías estrechamente relacionadas con la infección por H. pylori, se considera que es necesario buscar la infección y erradicarla, si está presente, en los pacientes con úlcera péptica activa (o antecedente de la misma si no conocemos el estado de erradicación en el paciente), linfoma MALT, cáncer gástrico temprano, dispepsia crónica, diagnóstico de púrpura, y usuarios crónicos de AINES, entre otros más debatidos ${ }^{7,8}$.

Conociendo la estrecha relación entre la infección por $H$. pylori y la aparición de cáncer 
gástrico, y sabiendo que estamos en una región del mundo con alta prevalencia de este tumor y una tasa elevada de resistencia de la bacteria al tratamiento antimicrobiano, este artículo tiene como objetivo tratar de dar respuesta a la pregunta ¿vale la pena afirmar que toda la población infectada requiere tratamiento, incluso si es asintomática?

\section{Método}

Se hizo una revisión bibliográfica de los últimos años, sobre los estudios que apoyan o rechazan el tratamiento universal de erradicación del $H$. pylori. La búsqueda se realizó en bases de datos como PubMed y LILACS, utilizando los términos DeCS descritos, además de opinión de expertos.

\section{Tratamiento universal: A favor}

La hipótesis más aceptada muestra que el H. pylori es el agente causal más importante involucrado en el desarrollo de cáncer gástrico. Aunque existen otros factores etiológicos para tener en cuenta en esta patología (consumo de cigarrillo y alcohol, dieta y genética), la infección por $H$. pylori explica hasta el $75 \%$ del cáncer gástrico a nivel mundial ${ }^{9}$. Con estos datos surge la pregunta si se debe erradicar esta infección en todos los individuos que la presenten, para disminuir la incidencia de cáncer gástrico.

Existe una revisión sistemática y metaanálisis reciente donde se analizaron seis ensayos clínicos aleatorizados controlados, y se concluyó que la erradicación del H. pylori reduce la incidencia de cáncer gástrico en individuos asiáticos asintomáticos ${ }^{10}$. Estas estrategias de erradicación deben ir de la mano de una mayor investigación para mejorar nuestro entendimiento sobre la etiología y los múltiples factores implicados en su desarrollo, además de contar con políticas de salud pública de prevención y promoción de estilos de vida saludable, para impactar en aquellos factores de riesgo modificables implicados en la fisiopatología de la enfermedad ${ }^{\text {II }}$. Debe anotarse que la erradicación del $H$. pylori deja de tener un papel clínicamente importante en el desarrollo de cáncer gástrico cuando ya existen lesiones preneoplásicas (gastritis atrófica y metaplasia intestinal), lo que refuerza la importancia de erradicar esta bacteria, antes de que estas lesiones se presenten en los pacientes, aun cuando estos sean asintomáticos.

$\mathrm{Al}$ analizar la literatura actual, y las publicaciones de trabajos realizados en países con alta incidencia de infección por $H$. pylori y cáncer gástrico, se encuentra el estudio retrospectivo de Leung W et al. ${ }^{8}$, llevado a cabo en Hong Kong, que pretendía evaluar los efectos del tratamiento del H. pylori en la incidencia de cáncer gástrico de su población. La búsqueda de los pacientes se realizó en la base de datos de la Autoridad Hospitalaria de Hong Kong en la que se identificaron los pacientes con infección por H. pylori que recibieron tratamiento de erradicación basado en claritromicina, entre los años 2003-2012. Se comparó el cáncer gástrico en esta cohorte con la incidencia esperada en la población general ajustado a la edad y el sexo, entre los años 2003 - 2014 (último año disponible) del Registro de Cáncer de Hong Kong. El resultado primario fue dado por la incidencia de desarrollo de cáncer gástrico en la cohorte tratada para la infección de H. pylori, con la incidencia de cáncer gástrico esperado en la población general.

Los análisis fueron realizados por grupos de edad a priori de menores de 40 años, entre $40-59$ años y de 60 años o más. De los 73.237 pacientes infectados con $H$. pylori que recibieron terapia de erradicación, $200(0,27 \%)$ desarrollaron cáncer gástrico durante una media de seguimiento de 7,6 años. Comparado con la población general, el riesgo de cáncer gástrico fue significativamente menor en los sujetos de 60 años o más que recibieron tratamiento para H. pylori, pero no en los grupos con pacientes más jóvenes ${ }^{8}$.

Cuando los resultados se analizan en base al tiempo en el que se erradicó el H. pylori (menos de 5 años, entre 5 - 9 años y más de Io años) el riesgo de cáncer gástrico fue significativamente menor que en la población general cuando se llevaban más de Io años después del tratamiento en el grupo de edad de 40 - 50 años y en el grupo de 
60 o más años en comparación con el grupo más joven. Un análisis secundario del estudio mostró además que un retraso en la erradicación (dado por el requerimiento de terapias adicionales en los pacientes, diferentes a la terapia triple basada en claritromicina) está asociado con un riesgo dos veces mayor de desarrollar cáncer gástrico con respecto a la población general (especialmente en el grupo entre los 40 - 59 años).

Aunque este estudio tiene algunas limitaciones como la falta de grupos control de pacientes no tratados similares a los grupos de pacientes tratados, esto debido principalmente a que las políticas de salud pública locales están encaminadas a tratar a todos los pacientes infectados, aun comparando el grupo de tratados frente a la población general, en la que se incluye población infectada o no por $H$. pylori, se logra demostrar una reducción significativa (mayor o igual a 60 años, tasa de incidencia estandarizada -TIE-: 0,42; $\mathrm{IC}_{95 \%}$ : 0,I8 - 0,84; 40-59 años: TIE: 0,32; $\mathrm{IC}_{95 \%} \%$ 0,08-0,88) en la incidencia de cáncer gástrico $(8, \mathrm{II})$.

Ha habido un interés creciente en la erradicación masiva del H. pylori para prevenir el cáncer gástrico. El beneficio de la erradicación varía en relación con el riesgo basal de este tipo de cáncer ${ }^{\text {Io }}$, el cual cambia ampliamente entre las regiones y las poblaciones ${ }^{12}$, por lo que el grado en que la erradicación masiva del $H$. pylori afectará la incidencia de cáncer gástrico continúa siendo un aspecto poco claro. Para ello, en el año 2016 se publicó una revisión sistemática de la literatura con un metaanálisis en la que se pretendía investigar los efectos de la erradicación del $H$. pylori en poblaciones con incidencias diferentes de cáncer gástrico. Se realizó una búsqueda de la literatura en diferentes bases de datos, en la que se incluyeron todos aquellos ensayos clínicos y estudios de cohorte realizados hasta mayo de 2015 , sin restricción del lenguaje, que compararan individuos que recibieron erradicación del $H$. pylori con aquellos que no, y su incidencia de cáncer gástrico, además de la recurrencia metacrónica después de la resección endoscópica de cáncer gástrico temprano. Encontraron que aquellos individuos que lograron erradicar la infección tuvieron una incidencia de cáncer gástrico menor que aquellos sin erradicación, con una tasa de incidencia de $0,54\left(\mathrm{IC}_{95 \%}: 0,46-0,65\right) \mathrm{sin}$ heterogeneidad significativa $\left(\mathrm{I}^{2}=0 \% ; \mathrm{p}=0,67\right)^{13}$.

Aquellos estudios que evaluaron la incidencia de cáncer luego de la resección endoscópica fueron agrupados de acuerdo con la incidencia global por terciles. En los estudios categorizados en el tercil intermedio (314,3 a 294I,2 por 100.000 años-persona) y el más alto (2970,0 a I0.256,4 por I00.000 años-persona) de incidencia basal de cáncer gástrico, las personas que recibieron tratamiento para H. pylori tuvieron una incidencia significativamente menor de cáncer gástrico en comparación con aquellos que no recibieron tratamiento, con una tasa de incidencia de 0,49 $\left(\mathrm{IC}_{95 \%}\right.$ : 0,38-0,64) y 0,45 $\left(\mathrm{IC}_{95 \%}: 0,32-0,64\right)$ respectivamente, pero sin un impacto estadísticamente significativo para aquellos estudios agrupados en los terciles bajos. Al comparar ambos resultados en el análisis por subgrupos se evidenció que el beneficio cuantitativo de la erradicación parecía mayor entre las personas después de una resección endoscópica (tasa de incidencia agrupada $=$ 0,$\left.46 ; \mathrm{IC}_{95 \%}: 0,35-0,60\right)$ que entre los individuos infectados asintomáticos (tasa de incidencia agrupada $\left.=0,62 ; \mathrm{IC}_{95 \%}: 0,49-0,79\right)$, pero la diferencia no fue significativa ( $\mathrm{p}=\mathrm{0}, \mathrm{I2}$ por meta regresión). Adicionalmente se observó que la tasa de incidencia de cáncer gástrico para la erradicación disminuyó por debajo de uno (factor protector), cuando la incidencia del mismo excedió 150 por I00.000 años-persona, lo que sugiere que en lugares de alta incidencia de cáncer gástrico existe un efecto benéfico de tratar a aquellos pacientes que incluso son asintomáticos, pues disminuye la tasa de incidencia global de cáncer ${ }^{13}$.

Si nos basamos en las recomendaciones actuales de acuerdo a los consensos más grandes y recientes acerca del manejo del $H$. pylori, como el Maastricht $\mathrm{V}^{4}$ publicado en el 20I7, todos los pacientes con evidencia de colonización por esta bacteria van a tener cambios en la mucosa y por ende riesgo de transformación maligna, independiente de los síntomas, ya que puede haber 
pacientes infectados, con cambios citológicos y asintomáticos, lo que le confiere la característica de carcinógeno tipo I para el cáncer gástrico. Por lo tanto, todos los pacientes en los que se demuestre la presencia de dicho microorganismo deberían tener un manejo de erradicación según la resistencia bacteriana local, ya que indudablemente representa un factor de riesgo para desarrollar patología maligna. Además, ha mostrado ser una medida costo-efectiva, sobre todo en países con alta incidencia de cáncer gástrico ${ }^{2,4}$.

Los autores que recomiendan erradicar el $H$. pylori en la población general, se basan en estudios como el de Sugano ${ }^{14}$, publicado en el 2019, una revisión sistemática de la literatura y un metaanálisis de 32 estudios, con más de 3 I.00o pacientes en total, para determinar si la erradicación de la bacteria contribuye con la disminución del cáncer gástrico. Dentro de los resultados presentados se encontró, con un OR de 0,46 ( IC $_{95 \%}$ : 0,38-0,54), que erradicar el $H$. pylori es un factor protector respecto al desarrollo de cáncer gástrico, sin embargo, esto va a depender de diferentes factores, como el país y su incidencia particular de malignidad, las condiciones basales de los pacientes y los períodos de seguimiento empleados. Por ejemplo, Japón y Corea, que son países con alta incidencia de cáncer gástrico, serían los países que más se beneficiarían de la erradicación.

También se logró demostrar la reducción de cáncer, en pacientes con úlcera péptica y gastritis crónica, asociado a la infección por el $H$. pylori, lo que ratifica la importancia de la erradicación de dicha bacteria en estadios tempranos, para lograr evitar la progresión a metaplasia, displasia y cáncer gástrico, siendo la metaplasia una lesión preneoplásica que no tiene retorno, a diferencia de los cambios por gastritis crónica. Asociado a esto existe un estudio publicado en 2018 por Choi et al., que demostró que la erradicación del $H$. pylori disminuye la incidencia de lesiones metacrónicas en los pacientes que van a resección endoscópica de cáncer temprano o de adenomas de alto grado comparado con el placebo ${ }^{15}$.

Todo lo expuesto previamente apoya que, en un país con alta incidencia de cáncer gástrico, como lo es Colombia, la erradicación del H. pylori debe hacerse en todo paciente en quien se identifique el mismo, independiente de si es sintomático o no, y si tiene comorbilidades o no, con el fin de disminuir la posibilidad de transformación de tejidos histológicos benignos en malignos en la mucosa gástrica.

\section{Tratamiento universal: En contra}

Si bien, diferentes estudios han demostrado la asociación que tiene el $H$. pylori con el cáncer gástrico, existe el debate de si es pertinente dar tratamiento o no a todas las personas infectadas con este microorganismo, incluso si son pacientes asintomáticos, debido a las altas tasas de resistencia antibiótica que ha desarrollado, lo que lleva a disminución de la eficacia del tratamiento, y alto riesgo de recurrencia a un año, el cual en Colombia es hasta del I8,I \% ${ }^{16}$.

Para comprender mejor esta problemática, debemos conocer cuál ha sido la relación que ha tenido el $H$. pylori a lo largo de la historia con el ser humano y su evolución en nuestra especie, logrando desarrollar procesos de adaptación únicos que le han permitido persistir a lo largo del tiempo, gracias a su capacidad de mutación y recombinación homóloga con otras cepas de su especie.

Hace aproximadamente 500 años, durante la colonización de América, nuevas cepas de $H$. pylori, $h p$ Europe y $h p$ África, fueron introducidas a nuestro territorio por los españoles y esclavos africanos respectivamente, desplazando así la cepa nativa, $h p$ Amerind, que habitaba en los indígenas nativos. Desde entonces este microorganismo ha evolucionado rápidamente, generando en nuestro país un subtipo de $H$. pylori propio, con características genéticas únicas, que le han conferido mayor virulencia por mutaciones en genes como el hor B, que codifica para una proteína de adhesión, y el gen vacA, que codifica para una citotoxina de gran importancia en la patogenia por infección de este microorganismo ${ }^{17}$.

Gracias a estas características propias de la bacteria, se piensa en reconsiderar el tratamiento 
indiscriminado a todos los pacientes con infección por $H$. pylori, debido a la resistencia a los antimicrobianos usualmente utilizados para su erradicación. En febrero de 2017 la Organización Mundial de la Salud publicó una lista de bacterias que requieren urgentemente nuevos antibióticos, donde el $H$. pylori resistente a la claritromicina aparece con prioridad alta, junto a bacterias como el Staphylococcus aureus resistente a la meticilina o con sensibilidad intermedia y resistencia a la vancomicina, y el Enterococcus faecium resistente a la vancomicina. Se confirma así la creciente resistencia del $H$. pylori en las diferentes latitudes y la importancia del uso cuidadoso de los antibióticos ${ }^{18}$.

La resistencia que existe ha llevado a tratamientos no exitosos en los intentos de erradicación, apareciendo cepas con resistencia múltiple a diferentes clases de antibióticos, e incluso, cepas multirresistentes (tres o más antibióticos) ${ }^{18}$, razón por la cual se han estudiado las causas de la resistencia del H. pylori. La causa más común son las mutaciones específicas, pero las transformaciones naturales junto con las bombas de flujo le confieren la multirresistencia. Las mutaciones comunes asociadas con la resistencia a la claritromicina son A2I43G y A2I42G, y menos frecuente A2I42C del gen ARNr 23S. También hay otras mutaciones relacionadas con la resistencia a otros antimicrobianos, como a las fluoroquinolonas con mutaciones en el gen gyrA, a las tetraciclinas en el gen del ARNr I6S, y a la rifabutina en el gen $r p o B$. La resistencia a la amoxicilina es supremamente rara, pero puede darse si hay mutaciones en los genes que alteran las proteínas de unión a la penicilina, junto a cambios en la permeabilidad de la membrana y las bombas de flujo ${ }^{19}$.

Las mutaciones previamente mencionadas, se deben al uso indiscriminado de antibióticos en nuestra población y a factores genéticos propios, lo que hace de la resistencia antibiótica una problemática importante en la actualidad. Preocupa que la resistencia al metronidazol y a la claritromicina, que son medicamentos de primera línea en nuestro país, son tan altas como del $25 \%$ y del $83 \%$ respectivamente ${ }^{20,21}$, al igual que el aumento de la resistencia a la levofloxacina, un medicamento de segunda línea en el tratamiento de la infección por H. pylori, también usado en infecciones respiratorias como la tuberculosis pulmonar. Para el 2009 se tenía una resistencia a la levofloxacina del II,8 \% y para el 2014 la resistencia creció a un $27,3 \%$. En estudios realizados en Colombia, se han aislado cepas, algunas de ellas únicas de nuestro país, con mutaciones específicas en el gen gyrA, que le confiere a la bacteria resistencia a este antibiótico ${ }^{22}$.

Para el año 20I6, en las poblaciones europeas había una tasa de resistencia a la claritromicina, metronidazol y ambos antibióticos del $20 \%$ al $35 \%, 29 \%$ al $60 \%$ y $23 \%$ respectivamente ${ }^{19}$. La presencia de multirresistencia (tres o más antibióticos) es menor al io \% en países como Austria, Portugal, Argentina, Francia y Bulgaria, y mayor del $40 \%$ en Perú ${ }^{23}$, siendo el patrón de multirresistencia más frecuente a claritromicina, metronidazol y quinolonas. Sin embargo, hay reporte de quíntuple y séxtuple resistencia en pacientes de China ${ }^{24}$, convirtiendo esto en un grave problema de salud pública en el mundo.

Se ha investigado la causa de la resistencia y la multirresistencia, encontrando que el principal factor es la presión de cepas resistentes por el consumo ambulatorio de antibióticos como macrólidos y fluoroquinolonas. Otro factor importante es la no adherencia a las guías de manejo para el H. pylori, que lleva al fallo terapéutico y posterior formación de resistencia ${ }^{25}$. También se ha evidenciado que la resistencia se debe a la coinfección de gérmenes con resistencias diferentes, llevando a que un régimen de tratamiento elimine las cepas sensibles a los antibióticos utilizados, pero aquellos resistentes perpetúan la infección ${ }^{19}$.

Además de la coinfección, se le ha conferido al H. pylori la capacidad de hetero-resistencia como posible causa de la multirresistencia, debido a que tiene la maquinaria genética para generar subpoblaciones de bacterias con mutaciones en diferentes genes, logrando la resistencia a distintas clases de antimicrobianos, sin embargo, se necesitan más estudios para definir con certeza esta capacidad del H. pylori ${ }^{26}$. 
Viendo los estudios locales, Roldán IJ et al., evaluaron la presencia de las mutaciones puntuales relacionadas con la resistencia a la claritromicina en la población que se sometía a endoscopia digestiva alta en un centro de la ciudad de $\mathrm{Me}$ dellín, y sobre un total de 396 pacientes, en 175 se encontró que tenían infección por H. pylori, y en 27 de ellos (I8,8 \%) encontraron la mutación en el gen del ARNr $23 \mathrm{~S}$ relacionado con resistencia a la claritromicina, lo que demuestra la alta prevalencia de la resistencia a la claritromicina del H. pylori, incluso en pacientes asintomáticos y en subpoblaciones con adecuada capacidad de acceso a servicios de salud ${ }^{27}$.

Lo anterior nos hace pensar que la infección y reinfección por el H. pylori es un problema multifactorial, en el cual intervienen además factores demográficos y socioeconómicos, en donde no solo basta la erradicación del microorganismo con manejo antibiótico, sino que se hace necesario también la creación e implementación de políticas de salud pública que vayan encaminadas en tratar aquellos determinantes reconocidos que están implicados en la propagación de la infección de este patógeno.

Como se ha mencionado anteriormente, las guías clínicas y los informes de consenso han recomendado sistemáticamente la detección y erradicación de $H$. pylori en poblaciones de alto riesgo ${ }^{7,28}$. Sin embargo, las preocupaciones sobre la implementación de un programa de detección y erradicación, no enfocado en poblaciones sanas y asintomáticas, aún persisten porque estos estudios incluyendo los metaanálisis han tenido varias limitaciones ${ }^{\text {Io }}$. Los hallazgos, originados en parte de estudios centrados en pacientes tratados por carcinoma gástrico en etapa temprana, no pueden aplicarse directamente a la población general. Además, las preocupaciones acerca de la evidencia obtenida de los estudios de cohortes incluyen sesgos de confusión y otras fuentes de sesgo ${ }^{29}$. Una revisión Cochrane reciente, evidenció que el único metaanálisis centrado en ensayos clínicos aleatorizados de sujetos sin carcinoma gástrico previo se basó en datos informados en resúmenes de congresos y utilizó enfoques estadísticos que pueden calcular intervalos de confianza falsamente estrechos cuando el número de estudios es pequeño y los eventos son $\operatorname{raros}^{10,29,30}$.

En el 20I7 Seta et al., publicaron un metaanálisis en el que se evaluó la eficacia de la erradicación del $H$. pylori en cuanto a la disminución de cáncer gástrico en población asintomática. Incluyeron siete ensayos clínicos aleatorizados, en los cuales se hizo un segundo análisis estadístico, partiendo de la premisa de que al evaluar los estudios solo por razón de riesgo (RR) estos tienden a exagerar el tamaño del efecto en comparación con el valor absoluto o el índice de diferencia, y haciendo caso a la recomendación de la Food and Drug Administration (FDA) en el 20II ${ }^{31}$, realizaron un nuevo análisis estadístico donde incluyeron estas razones, ya que solo cuatro de los estudios analizados los tuvieron en cuenta. Los resultados en términos de RR y $\mathrm{RD}$ (diferencia de riesgo) generales fueron 0,67 ( $\mathrm{IC}_{95 \%}$ : 0,48-0,95) y 0,00 ( $\mathrm{IC}_{95 \%}$ : 0,OI-0,00); número necesario para tratar: $125,5\left(\mathrm{IC}_{95 \%}: 70,0-800,9\right)$. Los resultados de RR presentados aquí no niegan la efectividad de la erradicación de $H$. pylori en la prevención del cáncer, concluyendo finalmente que en términos de riesgo absoluto (RA) y diferencia de riesgo $(R D)$, el tamaño del efecto fue menor y sin significancia estadística, por lo que se debe tener especial precaución al momento de promover medidas de erradicación basadas en la evidencia, especialmente si se habla de prevención primaria ${ }^{32}$.

Dadas estas limitaciones se hace necesario buscar estrategias estadísticas enfocadas en establecer la relación exacta, o al menos, con significancia, que ayude a dilucidar el interrogante sobre si realmente impacta la erradicación del H. pylori en la presentación de cáncer gástrico en pacientes asintomáticos. Es así como sale a la luz el metaanálisis de efecto aleatorio bayesiano ${ }^{33}$, el cual es una metodología flexible cada vez más utilizada, que da cuenta de la incertidumbre al combinar toda la evidencia externa disponible como la creencia inicial a través de la función de probabilidad, utilizando nuevos datos para 
actualizar la evidencia. Este estudio fue publicado en el 2019 e incluyó siete ensayos clínicos aleatorizados, cinco de China, uno de Japón y uno de nuestro país, evaluando 7303 adultos infectados con $H$. pylori sin carcinoma gástrico, confirmado por endoscopia digestiva superior. Las características de los estudios incluidos variaban en cuanto al tiempo de seguimiento, el tipo de confirmación y los algoritmos de erradicación adoptados, dos ensayos repitieron la terapia de primera línea para los no respondedores, dos no proporcionaron terapia de rescate y uno empleó la terapia de combinación dual. De los 7303 adultos incluidos solo se observaron $\mathrm{I} 38$ eventos de carcinoma gástrico. No se pudo observar el resultado primario en 369 sujetos que murieron por causas de carcinoma no gástrico y en 649 sujetos con datos perdidos.

Aunque las estimaciones resumidas mostraron una reducción del $35 \%$ en el riesgo de carcinoma gástrico asociado con la terapia de erradicación, el límite superior del $95 \%$ de los intervalos creíbles ( $\mathrm{ICr}_{95 \%}$ ) incluyó el I (efecto nulo), lo que sugiere efectos preventivos promedio no concluyentes de la terapia de erradicación en el riesgo de carcinoma gástrico $\left(\mathrm{HR}=0,65 ; \mathrm{ICr}_{95 \%}\right.$ O,4I a I,O; $\operatorname{IPr}_{95 \%}$ O,30 a I,33); I I = II \% $\left(\mathrm{ICr}_{95 \%}\right.$ I \% a $54 \%$ ); tau $=0,2 \mathrm{I}$ (baja certeza) ${ }^{33}$. La certeza de la evidencia se redujo por la imprecisión relacionada con el pequeño número de eventos $\mathrm{y}$ la aplicabilidad clínica limitada en la práctica actual, debido a las estrategias de erradicación insatisfactorias y la reinfección frecuente.

De lo anterior se puede concluir que este estudio con alto poder estadístico encontró solo una baja evidencia de que la erradicación de $H$. pylori pudo haber tenido mayor impacto que el tratamiento de control para reducir el desarrollo de carcinoma gástrico; esto resalta la incertidumbre en torno a la efectividad del programa desenfocado de prueba y erradicación, incluso en regiones de alto riesgo.

Un metaanálisis de efectos fijos publicado en 20I7, que utiliza la diferencia de riesgo como medida de resultado, informó una reducción de riesgo no significativa asociada con la terapia de erradicación ${ }^{32}$, lo que generó preocupación sobre las conclusiones de los metaanálisis anteriores y es congruente con los resultados obtenidos del metaanálisis bayesiano.

Es claro entonces que, debido a la insuficiente evidencia, es necesario llevar a cabo ensayos clínicos aleatorizados con mayor número de pacientes, rigurosamente planeados, que adopten metodologías de investigación estrictas, cuyo tema central sea la demostración de la verdadera eficacia de la terapia de erradicación, incluyendo el uso de estrategias de eliminación más efectivas, con una evaluación precisa de la respuesta, mejores terapias de rescate y un seguimiento meticuloso a largo plazo.

\section{Conclusión}

No hay pruebas aleatorias suficientes para apoyar o refutar la efectividad de la terapia de erradicación en la prevención del carcinoma gástrico en poblaciones de alto riesgo infectadas por H. pylori. Los ensayos clínicos aleatorizados, con una muestra significativa de pacientes adultos sanos infectados por $H$. pylori, que adopten metodologías de investigación más estrictas, proporcionarán evidencia sobre la verdadera eficacia de la erradicación exitosa. Mientras tanto, las estrategias de prevención deben enfocarse en políticas de salud pública que impliquen el mejoramiento de las condiciones sociosanitarias, así como el acceso temprano a los sistemas de salud ante la presencia de signos o síntomas tempranos, que permitan identificar poblaciones con lesiones precancerosas, permitiendo así impactar directamente sobre la presentación de carcinomas avanzados, su mortalidad y la calidad de vida.

Se deben fortalecer las estrategias de erradicación para lograr que estas sean más eficientes, ya sea al orientarlas con terapias antibióticas dirigidas por antibiogramas o haciendo seguimiento estrecho, en pro de mantener una adecuada adherencia y disminuir la tasa de resistencia bacteriana, para así poder tener un lugar más representativo en esta batalla, que para muchos se encuentra perdida. 


\section{Cumplimiento de normas éticas}

Consentimiento informado: Este estudio es una revisión de la literatura, y como tal no hay necesidad de un consentimiento informado.

Conflictos de interés: ninguno reportado por los autores.

Financiación: el presente trabajo fue autofinanciado por los autores.

\section{Referencias}

I. Cave DR, Go M, Cutler A, Goldstein J, Dunn B, Mobley $\mathrm{H}$, et al. Transmission and epidemiology of Helicobacter pylori. Am J Med. I996;IOO:I2S-I8S. https://doi.org/IO.IOI6/s0oo2-9343(96)80224-5

2. IARC Working Group on the Evaluation of Carcinogenic Risks to Humans. Schistosomes, liver flukes and Helicobacter pylori. Lyon, 7-I4 June I994. IARC Monogr Eval Carcinog Risks Hum. 1994;6I:I-24I.

3. Chey WD, Leontiadis GI, Howden CW, Moss SF. ACG Clinical Guideline: Treatment of Helicobacter pylori infection. Am J Gastroenterol. 20I7;112:212-39.

https://doi.org/IO.IO38/ajg.2016.563

4. Malfertheiner P, Megraud F, O'Morain C, Gisbert JP, Kuipers EJ, Axon A, et al. Management of helicobacter pylori infection - the Maastricht $\mathrm{V} /$ Florence consensus report. Gut. 20I7;66:6-30. https://doi.org/IO.II36/gutjnl-20I6-3I2288

5. Tonkic A, Tonkic M, Lehours P, Mégraud F. Epidemiology and diagnosis of Helicobacter pylori infection. Helicobacter. 20I2;17:I-8.

https://doi.org/IO.IIII/j.I523-5378.20I2.00975.x

6. Otero W, Trespalacios AA, Otero L, María T, Vallejo O, Torres Amaya M, et al. Guía de práctica clínica para el diagnóstico y tratamiento de la infección por Helicobacter pylori en adultos. Rev Colomb Gastroenterol. 2015;30(supl.I):17-33.

7. Sugano K, Tack J, Kuipers EJ, Graham DY, El-Omar EM, Miura S, et al. Kyoto global consensus report on Helicobacter pylori gastritis. Gut. 2015;64:1353-67. http://doi.org/IO.II36/gutjnl-20I5-309252

8. Leung WK, Wong IOL, Cheung KS, Yeung KF, Chan EW, Wong AYS, et al. Effects of Helicobacter pylori treatment on incidence of gastric cancer in older individuals. Gastroenterology. 2018;155:67-75. http://doi.org/IO.I053/j.gastro.2018.03.028

9. Oliveros R, Pinilla R, Facundo H, Sánchez R. Cáncer gástrico: una enfermedad prevenible. Estrategias para intervención en la historia natural. Rev Colomb Gastroenterol. 20I9;34:I77-89.

http://doi.org/IO.22516/25007440.394.
Io. Ford AC, Forman D, Hunt RH, Yuan Y, Moayyedi P. Helicobacter pylori eradication therapy to prevent gastric cancer in healthy asymptomatic infected individuals: Systematic review and meta-analysis of randomised controlled trials. BMJ. 20I4;348:g3I74.

https://doi.org/IO.II36/bmj.g3I74

II. Karimi P, Islami F, Anandasabapathy S, Freedman ND, Kamangar F. Gastric cancer: Descriptive epidemiology, risk factors, screening, and prevention. Cancer Epidemiol Biomarkers Prev. 20I4;23:700-I3. http://doi.org/IO.II58/IO55-9965.EPI-I3-IO57

I2. World Health Organization; International Agency for Research on Cancer. Helicobacter pylori Eradication as a Strategy for Preventing Gastric Cancer. Lyon, France: IARC Library Cataloguing in Publication Data; 2013. Fecha de consulta: 2I de enero de 2020. Disponible en: http://www.iarc.fr/en/publications/pdfs-online/wrk/ wrk8/index.php

I3. Lee YC, Chiang TH, Chou CK, Tu YK, Liao WC, Wu MS, et al. Association between Helicobacter pylori eradication and gastric cancer incidence: A Systematic review and meta-analysis. Gastroenterology. 2016;150:III3-24.e5. https://doi.org/IO.I053/j.gastro.2016.0I.028

I4. Sugano K. Effect of Helicobacter pylori eradication on the incidence of gastric cancer: a systematic review and meta-analysis. Gastric Cancer. 2019;22:435-45. https://doi.org/Io.IO07/sioi2o-or8-0876-o

I5. Choi IJ, Kook M-C, Kim Y-I, Cho S-J, Lee JY, Kim CG, et al. Helicobacter pylori therapy for the prevention of metachronous gastric cancer. N Engl J Med. 2018;378:I085-95. http://doi.org/IO.IO56/NEJMoaI708423

I6. Morgan DR, Torres J, Sexton R, Herrero R, Salazar-Martínez E, Greenberg ER, et al. Risk of recurrent helicobacter pylori infection I year after initial eradication therapy in 7 Latin American communities. JAMA. 2013;309:578-86. http://doi.org/IO.IOoI/jama.20I3.3II

I7. Gutiérrez-Escobar AJ, Trujillo E, Acevedo O, Bravo MM. Phylogenomics of Colombian Helicobacter pylori isolates. Gut Pathog. 2017;9:52.

https://doi.org/IO.II86/sI3099-OI7-O2OI-I

I8. World Health Organization. Global priority list of antibiotic-resistant bacteria to guide research, discovery, and development of new antibiotics. Ginebra: WHO; 20I7. p.7. Fecha de consulta: 2I de enero de 2020. Disponible en: https://www.who.int/medicines/publications/ global-priority-list-antibiotic-resistant-bacteria/en/

19. Boyanova L, Hadzhiyski P, Kandilarov N, Markovska R, Mitov I. Multidrug resistance in Helicobacter pylori: current state and future directions. Expert Rev Clin Pharmacol. 2019;12:909-I5. https://doi.org/IO.IO80/I75I2433.2019.I654858

20. Camargo MC, García A, Riquelme A, Otero W, Camargo CA, Hernández-García T, et al. The problem 
of helicobacter pylori resistance to antibiotics: A systematic review in latin America. Am J Gastroenterol. 20I4;I09:485-95. https://doi.org/IO.IO38/ajg.20I4.24

2I. Otero W, Gómez M, Otero L, Trespalacios A. Helicobacter pylori: ¿cómo se trata en el 20I8?. Rev gastroenterol Perú. 2018;38:54-63.

22. Trespalacios-Rangel AA, Otero W, Arévalo-Galvis A, Poutou-Piñales RA, Rimbara E, Graham DY. Surveillance of levofloxacin resistance in helicobacter pylori isolates in Bogotá-Colombia (2009-20I4). PLoS One. 20I6;II:eoI600o7.

https://doi.org/Io.I371/journal.pone.0I600o7

23. Boehnke KF, Valdivieso M, Bussalleu A, Sexton R, Thompson KC, Osorio S, et al. Antibiotic resistance among Helicobacter pylori clinical isolates in Lima, Peru. Infect Drug Resist. 20I7;IO:85-90. https://doi.org/IO.2I47/IDR.SI23798

24. Zhang YX, Zhou LY, Song ZQ, Zhang JZ, He LH, Ding Y. Primary antibiotic resistance of Helicobacter pylori strains isolated from patients with dyspeptic symptoms in Beijing: A prospective serial study. World J Gastroenterol. 20I5;2I:2786-92.

https://doi.org/IO.3748/wjg.v2I.i9.2786

25. Megraud F, Coenen S, Versporten A, Kist M, Lopez-Brea M, Hirschl AM, et al. Helicobacter pylori resistance to antibiotics in Europe and its relationship to antibiotic consumption. Gut. 2013;62:34-42.

https://doi.org/Io.II36/gutjnl-2012-302254

26. Rizvanov AA, Haertlé T, Bogomolnaya L, Abadi ATB. Helicobacter pylori and its antibiotic heteroresistance: A neglected issue in published guidelines. Frontiers in Microbiology. 20I9;IO. Fecha de consulta: 2I de enero de 2020. Disponible en:

https://repository.kpfu.ru/?p_id=206583

27. Roldán IJ, Castaño R, Navas MC. Mutaciones del gen ARN ribosómico $23 \mathrm{~S}$ de Helicobacter pylori asociadas con resistencia a claritromicina en pacientes atendidos en una unidad de endoscopia de Medellín, Colombia. Biomédica. 2019;39:117-29.

https://doi.org/I0.7705/biomedica.v39i4.4377

28. Kim SG, Jung HK, Lee HL, Jang JY, Lee H, Kim CG, et al. Guidelines for the diagnosis and treatment of Helicobacter pylori infection in Korea, 2013 revised edition. J Gastroenterol Hepatol. 20I4;29:137I-86. https://doi.org/Io.IIII/jgh.I2607

29. Doorakkers E, Lagergren J, Engstrand L, Brusselaers N. Eradication of Helicobacter pylori and gastric cancer: A systematic review and meta-analysis of cohort studies. J Natl Cancer Inst. 20I6;I08:pii:djwi32. https://doi.org/Io.Io93/jnci/djwi32

30. Falagas ME, Rosmarakis ES. Clinical decision-making based on findings presented in conference abstracts: Is it safe for our patients? Eur Heart J. 2006;27:2038-9. https://doi.org/IO.I093/eurheartj/ehlı75

3I. Fischhoff B, Brewer NT. Communicating Risks and Benefits: An Evidence-Based User's Guide. In: Fagerlin A, Peters E (ed.). Chapter 7: Quantitative Information. FDA: Montgomery, Maryland; 20II. P.53-64.

32. Seta T, Takahashi Y, Noguchi Y, Shikata S, Sakai T, Sakai $\mathrm{K}$, et al. Effectiveness of Helicobacter pylori eradication in the prevention of primary gastric cancer in healthy asymptomatic people: A systematic review and meta-analysis comparing risk ratio with risk difference. PLoS One. 20I7;I2:eoI8332I. https://doi.org/I0.I37I/journal.pone.oI8332I

33. Terasawa T, Hamashima C, Kato K, Miyashiro I, Yoshikawa T, Takaku R, et al. Helicobacter p ylori eradication treatment for gastric carcinoma prevention in asymptomatic or dyspeptic adults: Systematic review and Bayesian meta-analysis of randomised controlled trials. BMJ Open. 2019;9:e026002.

https://doi.org/IO.II36/bmjopen-2018-026002 